\title{
Nonlinear Viscoelasticity of Concentrated Solution of Rod-like Polymers
}

\author{
Nobu Y. KUZUU and Masao DoI \\ Department of Physics, Faculty of Science, Tokyo Metropolitan University, \\ Fukazawa 2-1-1, Setagaya-ku, Tokyo 158, Japan.
}

(Received June 21, 1980)

\begin{abstract}
The nonlinear viscoelasticity of concentrated solutions of rod-like polymers in the isotropic phase was studied theoretically based on the molecular diffusion equation of DoiEdwards. Rheological functions were calculated for both shear and elongational flows in a steady, and transient state. The viscoelastic properties of this system were found to be very similar to those of flexible polymers despite the difference in molecular shape and the mechanism of Brownian motion: e.g., shear thinning, normal stresses, and stress overshoot were predicted for rod-like polymers.
\end{abstract}

KEY WORDS Concentrated Solution / Rod-like Polymers / Nonlinear

Viscoelasticity / Transient Flow / Simple Shear Flow / Elongational Flow /

The viscoelastic properties of concentrated solution of rod-like polymers are very important from the stand point of industrial application. ${ }^{1}$ In the past ten years, several experimental and theoretical studies have been reported. ${ }^{2-6}$

Though the transient flow and elongational flow are important in fiber or film formation processes, the studies so far carried out are mostly concerned with the linear viscoelasticity, or the steady-state simple shear flow. In this paper, we study theoretically the nonlinear viscoelasticity of this system under simple shear and elongational flows in both the steady state, and the transient state. We show that the viscoelastic properties of this system are qualitatively very similar to those of flexible polymers.

The Brownian motion of rigid rod-like polymers in a concentrated solution in the isotropic phase has been discussed by Doi $^{7}$ and Doi-Edwards, ${ }^{8,9}$ and the formula for calculating the stress tensor for a given velocity gradient is presented in ref 9 .

According to Doi-Edwards, ${ }^{9}$ given the history of the velocity gradient, the stress at time $t$ can be calculated as follows.

Let $\boldsymbol{u}$ be the unit vector parallel to a rod and $f(u ; t)$ be its orientational-distribution function at time $t$. The distribution function $f$ satisfies the following diffusion equation.

$$
\frac{\partial f}{\partial t}=\frac{\partial}{\partial \boldsymbol{u}} \cdot\left(\tilde{\tilde{D}_{\mathrm{r}}} \frac{\partial f}{\partial \boldsymbol{u}}\right)-\frac{\partial}{\partial \boldsymbol{u}} \cdot\{[\boldsymbol{\kappa} \cdot \boldsymbol{u}-(\boldsymbol{u} \cdot \boldsymbol{\kappa} \cdot \boldsymbol{u}) \boldsymbol{u}] f\}
$$

where $\boldsymbol{\kappa}$ is the transpose of a velocity gradient tensor and $\tilde{D}_{\mathrm{r}}(\boldsymbol{u} ;[f])$ the rotational-diffusion coefficient give by

$$
\tilde{D}_{\mathrm{r}}(\boldsymbol{u} ;[f])=D_{\mathrm{r}}\left[\frac{4}{\pi} \int \mathrm{d} \Omega^{\prime} f\left(\boldsymbol{u}^{\prime} ; t\right) \sin \left(\boldsymbol{u}, \boldsymbol{u}^{\prime}\right)\right]^{-2}
$$

in which $D_{\mathrm{r}}$ is the diffusion coefficient when the rods are distributed isotropically, and $\left(\boldsymbol{u}, \boldsymbol{u}^{\prime}\right)$ indicates the angle between $\boldsymbol{u}$ and $\boldsymbol{u}^{\prime}$. The stress tensor $\boldsymbol{\sigma}$ is calclated by ${ }^{9}$

$$
\boldsymbol{\sigma}=3 c k_{\mathrm{B}} T\langle\boldsymbol{u} \boldsymbol{u}\rangle+P \mathbf{1}
$$

here $c$ is the number of rods per unit volume, $k_{\mathrm{B}}$ the Boltzmann constant, $T$ the absolute temperature, $P$ the hydrostatic pressure, $\boldsymbol{u} \boldsymbol{u}$ indicates the diadic product of $\boldsymbol{u}$ and $\boldsymbol{u}$, and $\mathbf{1}$ the unit tensor. The average is taken over the orientational-distribution function determined from eq 1.

Since $\tilde{D}_{\mathrm{r}}$ depends on $\boldsymbol{u}$, solving the diffusion equation 1 is a difficult task even with the aid of computer. Therefore as in ref $9 \widetilde{D}_{\mathrm{r}}$ has been replaced by an effective diffusion coefficient $\bar{D}_{\mathrm{r}}$ defined by

$$
\bar{D}_{\mathrm{r}}=D_{\mathrm{r}}\left[\frac{4}{\pi} \iint \mathrm{d} \Omega \mathrm{d} \Omega^{\prime} f(\boldsymbol{u} ; t) f\left(\boldsymbol{u}^{\prime} ; t\right) \sin \left(\boldsymbol{u}, \boldsymbol{u}^{\prime}\right)\right]^{-2}
$$


which depends on $f$ but not on $\boldsymbol{u}$.

\section{ELONGATIONAL FLOW}

First we discuss the elongational flow, the velocity gradient tensor of which is given by

$$
\boldsymbol{\kappa}(t)=\left[\begin{array}{rrr}
-1 & 0 & 0 \\
0 & -1 & 0 \\
0 & 0 & 2
\end{array}\right] \frac{\dot{\varepsilon}(t)}{2}
$$

where $\dot{\varepsilon}(t)$ is the elongational rate. Since this flow has uniaxial symmetry, the diffusion equation 1 is written, in terms of the polar coordinate

$$
\begin{aligned}
& x_{1}=r \sin \theta \cos \varphi \\
& x_{2}=r \sin \theta \sin \varphi \\
& x_{3}=r \cos \theta
\end{aligned}
$$

as follows.

$$
\frac{\partial f}{\partial t}=\bar{D}_{\mathrm{r}} \frac{\partial}{\partial \zeta}\left[\left(1-\zeta^{2}\right)\left(\frac{\partial f}{\partial \zeta}-\overline{\alpha \zeta} f\right)\right]
$$

in which

$$
\bar{\alpha} \equiv \frac{3 \dot{\varepsilon}}{2 \bar{D}_{\mathrm{r}}}
$$

and $\zeta=\cos \theta$. Since $\bar{D}_{\mathrm{r}}$ depends on $f(\boldsymbol{u} ; t), \bar{\alpha}$ is not proportional to $\alpha$, but consistently determined from eq $2^{\prime}$ and 7 as

$\bar{\alpha}=\alpha\left[\frac{4}{\pi} \iint \mathrm{d} \Omega \mathrm{d} \Omega^{\prime} f(\boldsymbol{u} ; t) f\left(\boldsymbol{u}^{\prime} ; t\right) \sin \left(\boldsymbol{u}, \boldsymbol{u}^{\prime}\right)\right]^{-2}$

with

$$
\alpha \equiv \frac{3 \dot{\varepsilon}}{2 D_{\mathrm{r}}}
$$

\section{Steady-State Elongational Flow}

In the steady state, the diffusion equation 6 becomes

$$
0=\frac{\partial}{\partial \zeta}\left[\left(1-\zeta^{2}\right)\left(\frac{\partial f}{\partial \zeta}-\bar{\alpha} \zeta f\right)\right]
$$

This equation can easily be solved as

$$
f=C \exp \left(\bar{\alpha} \zeta^{2} / 2\right)
$$

where $C$ is a normalization factor.

$$
C^{-1}=2 \pi \int_{-1}^{1} \exp \left(\bar{\alpha}^{2} / 2\right) \mathrm{d} \zeta
$$

We express the result by the Trouton viscosity, defined by

$$
\bar{\eta}(\dot{\varepsilon})=\left[\sigma_{33}(\dot{\varepsilon})-\sigma_{11}(\dot{\varepsilon})\right] / \dot{\varepsilon}
$$

From eq 3,11 , and 13, the steady-state Trouton viscosity $\bar{\eta}(\dot{\varepsilon})$ is given by

$$
\bar{\eta}(\dot{\varepsilon})=\frac{3 c k_{\mathrm{B}} T}{20 D_{\mathrm{r}} \alpha}\left[\frac{3 \int_{0}^{1} \zeta^{2} \exp \left(\bar{\alpha} \zeta^{2} / 2\right) \mathrm{d} \zeta}{\int_{0}^{1} \exp \left(\bar{\alpha} \zeta^{2} / 2\right) \mathrm{d} \zeta}-1\right]
$$

In particular, when $\dot{\varepsilon}=0$

$$
\bar{\eta}(0)=\frac{3 c k_{\mathrm{B}} T}{10 D_{\mathrm{r}}}
$$

This is three times larger than the shear viscosity at the zero shear rate (see eq 44). For small value of $\alpha$, eq 14 can be expanded into power series of $\bar{\alpha}$ as

$\bar{\eta}(\dot{\varepsilon}) / \bar{\eta}(0)=\frac{1}{\alpha}\left(\bar{\alpha}+\frac{1}{21} \bar{\alpha}^{2}-\frac{1}{315} \bar{\alpha}^{3}-\frac{1}{2077} \bar{\alpha}^{4}+\cdots\right)$

In this case, eq 8 may also be expanded into a power series of $\bar{\alpha}$ as

$$
\alpha=\bar{\alpha}+\frac{1}{180} \bar{\alpha}^{3}+\frac{1}{1890} \bar{\alpha}^{4}+\cdots
$$

Equations 16 and 17 clearly determine the $\alpha$ $\left(=3 \dot{\varepsilon} / 2 D_{\mathrm{r}}\right)$ dependence of $\bar{\eta}(\dot{\varepsilon})$.

For larges value of $\alpha$, we use the asymptotic expansion

$$
\bar{\eta}(\dot{\varepsilon}) / \bar{\eta}(0) \sim \frac{15}{\alpha}\left(1-\frac{3}{\bar{\alpha}}-\frac{21}{\bar{\alpha}^{2}}-\frac{114}{\bar{\alpha}^{3}}-\cdots\right)
$$

and eq 8 becomes

$$
\bar{\alpha} \simeq 2.26 \sqrt{\alpha}
$$

The derivation of eq 18 and 19 is given in Appendix.

In Figure $1, \bar{\eta}(\dot{\varepsilon})$ is plotted against $\alpha$. The Trouton viscosity decreases almost monotonically with the elongational rate. Actually, the curve has a very weak peak at $\alpha \approx 2.5$, as can be seen from eq 16 , but this peak is negligible in the doubly logarithmic plot.

\section{Stress Growth in the Start of Elongational Flow}

Next, we consider the stress growth when an elongational flow starts at $t=0$. The development of the elongational rate may be represented by 


$$
\dot{\varepsilon}(t)= \begin{cases}0 & \text { for } t<0 \\ \dot{\varepsilon}(=\text { constant }) & \text { for } \quad t>0\end{cases}
$$

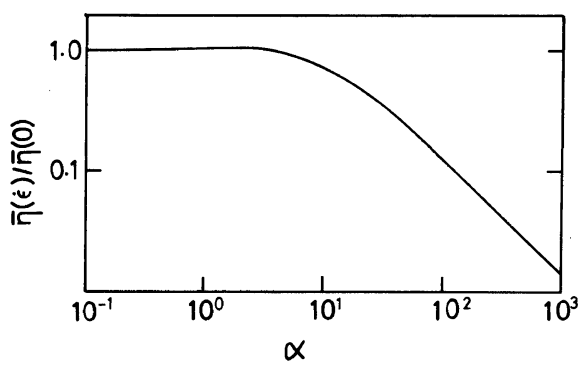

Figure 1. Normalized Trouton viscosities $\bar{\eta}(\dot{\varepsilon})$ are plotted against $\alpha=3 \dot{\varepsilon} / 2 D_{\mathrm{r}}$.

Since the orientational-distribution function does not depend on $\varphi$, it can be expanded into the Legendre polynomials

$$
f(\theta, \varphi)=\sum_{l=0}^{\infty} a_{l}(t) P_{l}(\cos \theta) / 4 \pi
$$

Because of the symmetry of the elongational flow, terms with odd $l$ do not appear. Substituting eq 21 into eq 6 , we have

$$
\begin{aligned}
\frac{\mathrm{d} a_{l}}{\mathrm{~d} t}= & -D_{\mathrm{r}} \alpha l(l+1)\left\{\left[\frac{1}{\bar{\alpha}}-\frac{1}{(2 l+3)(2 l-1)}\right] a_{l}\right. \\
& \left.+\frac{l+2}{(2 l+5)(2 l-1)} a_{l+2}-\frac{l-1}{(2 l-3)(2 l-1)} a_{l-2}\right\}
\end{aligned}
$$

From eq 21, eq 8 becomes

$$
\bar{\alpha}=\alpha\left\{1-2 \sum_{n=1}^{\infty} \frac{2 n-1}{(2 n+2)(4 n+1)}\left[\frac{(2 n-3) ! !}{(2 n) ! !}\right]^{2}\left|a_{2 n}\right|^{2}\right\}^{2}
$$

From the normalization condition

$$
\int f(u ; t) \mathrm{d} \Omega=1
$$

$a_{0}$ is determined as

$$
a_{0}=1
$$

Equation 22 was solved numerically by truncating $a_{l}$ with $l \geqq l^{*}\left(l^{*}\right.$ was taken as $12-16$ ).

We define the stress-growth function $\bar{\eta}^{+}(\dot{\varepsilon} ; t)$ as

$$
\bar{\eta}^{+}(\dot{\varepsilon} ; t)=\left[\sigma_{33}(\dot{\varepsilon} ; t)-\sigma_{11}(\dot{\varepsilon} ; t)\right] / \dot{\varepsilon}
$$

The function $\bar{\eta}^{+}(\dot{\varepsilon} ; t)$ is related to $a_{2}$ as

$$
\bar{\eta}^{+}(\dot{\varepsilon} ; t)=\frac{3 \bar{\eta}(0)}{\alpha} a_{2}
$$

The calculation results are shown in Figure 2. The stress grows monotonically and reaches a steadystate value.

The same results are shown in Figure 3, where the stress $\sigma_{33}(\dot{\varepsilon} ; t)$ is plotted against the elongational ratio

$$
\lambda=\mathrm{e}^{\dot{\varepsilon} t}
$$

In Figure 3, the value of $\lambda$ at which the stress reaches a steady-state value is almost independent of $\dot{\varepsilon}$.

The curve denoted by $\infty$ indicates the case in which $\alpha$ is infinity. This curve is calculated as follows. If $\alpha=\infty$, the effect of the rotational Brownian motion is negligible, and the orientation vector $\boldsymbol{u}$ of the rods changes according to the macroscopic flow. Suppose that the material point $\boldsymbol{r}$ at time $t=0$ is transformed to a point $\boldsymbol{E} \cdot \boldsymbol{r}$ at time $t$ by the macroscopic deformation. The orientation vector

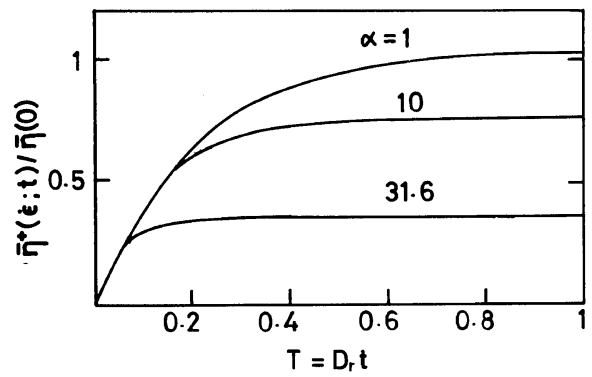

Figure 2. Growth of the Trouton viscosity when an elongational flow is started at $t=0$ with elongational rate $\alpha$.

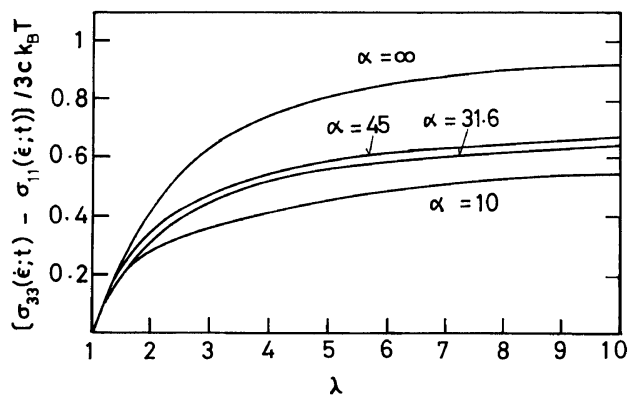

Figure 3. Plots of the stresses $\sigma_{33}(\dot{\varepsilon} ; t)-\sigma_{11}(\dot{\varepsilon} ; t)$ against the elongational ratio $\lambda=\mathrm{e}^{\dot{\varepsilon} t}$. 
$\boldsymbol{u}$ of the rod then changes to

$$
\boldsymbol{u}^{\prime}=\frac{\boldsymbol{E} \cdot \boldsymbol{u}}{|\boldsymbol{E} \cdot \boldsymbol{u}|}
$$

Since the rods are in isotropic distribution at $t=0$, the stress tensor is calculated by

$$
\boldsymbol{\sigma}^{\infty}=3 c k_{\mathrm{B}} T\left\langle\frac{(\boldsymbol{E} \cdot \boldsymbol{u})(\boldsymbol{E} \cdot \boldsymbol{u})}{|\boldsymbol{E} \cdot \boldsymbol{u}|^{2}}\right\rangle_{0}+P \mathbf{1}
$$

where $\langle\cdots\rangle_{0}$ indicates the average throughout the isotropic orientation

$$
\langle\cdots\rangle_{0}=\frac{1}{4 \pi} \int \mathrm{d} \Omega \cdots
$$

In the uniaxial elongation, $\boldsymbol{E}$ is represented in Cartesian coordinates as

$$
\boldsymbol{E}=\left[\begin{array}{lll}
\lambda^{-1 / 2} & 0 & 0 \\
0 & \lambda^{-1 / 2} & 0 \\
0 & 0 & \lambda
\end{array}\right]
$$

From eq 30 and 32, the stress for $\alpha=\infty$ is calculated as

$$
\begin{gathered}
\sigma_{33}-\sigma_{11}=3 c k_{\mathrm{B}} T \int \frac{\mathrm{d} \Omega}{4 \pi} \frac{\lambda^{2} u_{3}{ }^{2}-\lambda^{-1} u_{1}{ }^{2}}{\lambda^{2} u_{3}{ }^{2}+\lambda^{-1}\left(u_{1}{ }^{2}+u_{2}{ }^{2}\right)} \\
=\frac{9 c k_{\mathrm{B}} T}{2} \frac{\lambda^{3}}{\lambda^{3}-1}\left(1-\frac{\tan ^{-1} \sqrt{\lambda^{3}-1}}{\sqrt{\lambda^{3}-1}}\right)-\frac{1}{2} \\
(\lambda>0)
\end{gathered}
$$

\section{SIMPLE SHEAR FLOW}

In the simple shear flow, the velocity-gradient tensor $\boldsymbol{\kappa}(t)$ is given in Cartesian coordinates as

$$
\boldsymbol{\kappa}(t)=\left[\begin{array}{lll}
0 & \kappa(t) & 0 \\
0 & 0 & 0 \\
0 & 0 & 0
\end{array}\right]
$$

where $\kappa(t)$ is the shear rate. If we choose the polar coordinates as

$$
\begin{aligned}
& x_{1}=r \sin \theta \cos \varphi \\
& x_{2}=r \cos \theta \\
& x_{3}=r \sin \theta \sin \varphi
\end{aligned}
$$

(which is different from that in the preceding section), the diffusion equation becomes

$$
\frac{\partial f}{\partial t}=-\bar{D}_{\mathrm{r}} \hat{L}^{2} f-\kappa(t) \hat{\Gamma} f
$$

in which

$$
-\hat{L}^{2} \equiv \frac{1}{\sin \theta} \frac{\partial}{\partial \theta}\left(\sin \theta \frac{\partial}{\partial \theta}\right)+\frac{1}{\sin ^{2} \theta} \frac{\partial^{2}}{\partial \varphi^{2}}
$$

and

$$
\hat{\Gamma} \equiv \cos ^{2} \theta \cos \varphi \frac{\partial}{\partial \varphi}+\cot \theta \frac{\partial}{\partial \varphi}-3 \sin \theta \cos \theta \cos \varphi
$$

In this flow, there are three independent components of the stress tensor: Shear stress $\sigma_{12}$, the first normal stress difference $N_{1}=\sigma_{11}-\sigma_{22}$ and the second normal stress difference $N_{2}=\sigma_{22}-\sigma_{33}$.

The steady state of this flow was analysed by DoiEdwards, ${ }^{9}$ and the results are replotted in Figure 4.

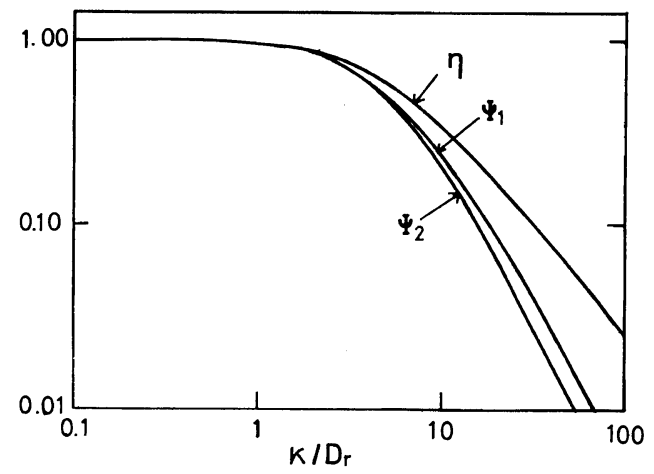

Figure 4. Normalized viscosity $\eta(\kappa)$, the first normal stress coefficient $\Psi_{1}(\kappa)$ and the second normal stress coefficient $\Psi_{2}(\kappa)$ (after ref 2 ).

Here the viscosity $\eta(\kappa)$, the first and the second normal stress coefficients $\Psi_{1}(\kappa)$ and $\Psi_{2}(\kappa)$ are defined by

$$
\begin{aligned}
& \sigma_{12}=\eta(\kappa) \kappa \\
& N_{1}=\Psi_{1}(\kappa) \kappa^{2} \\
& N_{2}=\Psi_{2}(\kappa) \kappa^{2}
\end{aligned}
$$

\section{Small Applitude Oscillation}

Consider a small amplitude oscillation

$$
\kappa(t)=\kappa_{0} \operatorname{Re} \mathrm{e}^{i \omega t}
$$

For small $\kappa_{0}$, the shear stress is perturbed linearly in $\kappa_{0}$, but the normal stress differences are quadratic in 
$\kappa_{0}$.

The following quantities are taken into consideration: the complex viscosity $\eta^{*}(\omega)$, the complex normal stress coefficients $\Psi_{1}^{*}(\omega)$ and $\Psi_{2}^{*}(\omega)$ and these are defined by

$$
\begin{aligned}
\sigma(t) & =\operatorname{Re}\left[\eta^{*}(\omega) \kappa_{0} \mathrm{e}^{i \omega t}\right] \\
N_{1}(t) & =\Psi_{1}^{d}(\omega) \kappa_{0}^{2}+\operatorname{Re}\left[\Psi_{1}^{*}(\omega) \kappa_{0}^{2} \mathrm{e}^{2 i \omega t}\right] \\
N_{2}(t) & =\Psi_{2}^{d}(\omega) \kappa_{0}^{2}+\operatorname{Re}\left[\Psi_{2}^{*}(\omega) \kappa_{0}^{2} \mathrm{e}^{2 i \omega t}\right]
\end{aligned}
$$

To calculate the functions defined by eq 41 , we expand the distribution function $f$ into the power series of $\kappa(t)$

$$
f=\frac{1}{4 \pi} \operatorname{Re}\left[1+\kappa_{0} \psi_{1} \mathrm{e}^{i \omega t}+\kappa_{0}^{2}\left(\psi_{2}^{(0)}+\mathrm{e}^{2 i \omega t} \psi_{2}^{(0)}\right)+\cdots\right]
$$

and use the usual perturbation method with respect to $\kappa_{0}$. As result, we obtain

$$
\begin{gathered}
\eta^{*}(\omega) / \eta(0)=\frac{1}{1+i \omega \tau_{\mathrm{r}}} \\
\Psi_{1}^{\mathrm{d}}(\omega) / \Psi_{1}(0)=\Psi_{2}^{\mathrm{d}}(\omega) / \Psi_{2}(0)=\frac{1}{2\left[1+\left(\omega \tau_{\mathrm{r}}\right)^{2}\right]} \\
\Psi_{1}^{*}(\omega) / \Psi_{1}(0)=\Psi_{2}^{*}(\omega) / \Psi_{2}(0)=\frac{1}{2\left(1+i \omega \tau_{\mathrm{r}}\right)\left(1+2 i \omega \tau_{\mathrm{r}}\right)}
\end{gathered}
$$

in which $\tau_{\mathrm{r}} \equiv 1 / 6 D_{\mathrm{r}}$ and $\eta(0), \Psi_{1}(0)$, and $\Psi_{2}(0)$ are the material functions for the steady flow at zeroshear rate ${ }^{9}$

$$
\eta(0)=\frac{c k_{\mathrm{B}} T}{10 D_{\mathrm{r}}}, \quad \Psi_{1}(0)=\frac{c k_{\mathrm{B}} T}{30 D_{\mathrm{r}}}, \quad \Psi_{2}(0)=-\frac{c k_{\mathrm{B}} T}{105 D_{\mathrm{r}}}
$$

These variables may be split into real and imaginary parts:

$$
\begin{gathered}
\eta^{*}(\omega)=\eta^{\prime}(\omega)-i \eta^{\prime \prime}(\omega) \\
\Psi_{1}^{*}(\omega)=\Psi_{1}^{\prime}(\omega)-i \Psi_{1}^{\prime \prime}(\omega) \\
\Psi_{2}^{*}(\omega)=\Psi_{2}^{\prime}(\omega)-i \Psi_{2}^{\prime \prime}(\omega)
\end{gathered}
$$

These real and imaginary parts and the absolute value of the complex viscosity are shown in Figure 5 .

In flexible polymers, there is a useful empirical relation, called the Cox-Mertz rule, which states that the magnitude of the complex viscosity is comparable with the steady-state viscosity at equal values of frequency and shear rate, i.e.,

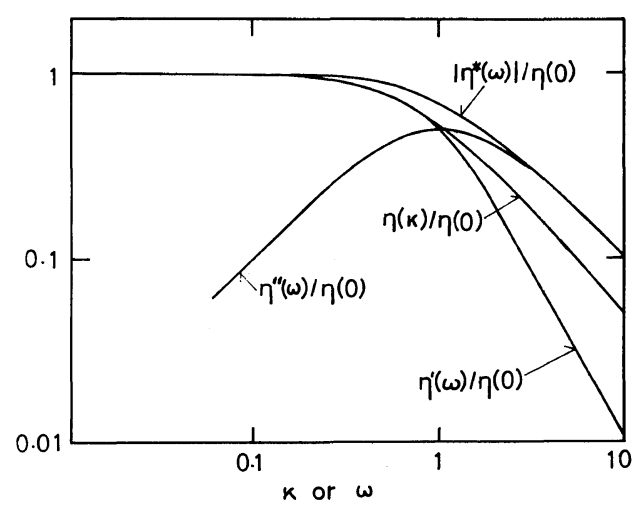

Figure 5. Plots of $\eta^{\prime}(\omega), \eta^{\prime \prime}(\omega)$, and $\eta^{*}(\omega)$ against $\omega$. The steady state viscosity $\eta(\kappa)$ is also plotted.

$$
\left.\left|\eta^{*}(\omega)\right| \approx \eta(\kappa)\right|_{\kappa=\omega}
$$

In Figure 5, $\eta^{*}(\omega)$ and $\eta(\omega)$ are compared at the same value of $\kappa$ and $\omega$. Though they do not match perfectly, there is considerable correlation between them.

\section{Transient Flows}

For the transient flow, a numerical calculation is needed to solve the diffusion equation 36 . The method for this is discussed in the subsection of this paper.

We expand $f$ into the spherical harmonics $Y_{l m}(\theta, \varphi)$ as $^{9}$

$$
f(\theta, \varphi ; t)=\sum_{l=0}^{\infty} \sum_{m=0}^{l} \mid l, m>b_{l m}
$$

with

$$
\mid l, m>= \begin{cases}Y_{l m}(\theta, \varphi) & \text { for } m=0 \\ \frac{1}{\sqrt{2}}\left[Y_{l m}(\theta, \varphi)+(-)^{m} Y_{l,-m}(\theta, \varphi)\right] \\ & \text { for } 1 \leqq m \leqq l\end{cases}
$$

Substituting eq 46 into eq 35 , we have

$$
\begin{aligned}
\frac{\mathrm{d} b_{l m}}{\mathrm{~d} t}= & -D_{\mathrm{r}} l(l+1) X b_{l m} \\
& -\kappa(t) \sum_{l^{\prime}=0}^{\infty} \sum_{m^{\prime}=0}^{l^{\prime}}\left\langle l, m|\hat{\Gamma}| l^{\prime}, m^{\prime}\right\rangle b_{l^{\prime} m^{\prime}}
\end{aligned}
$$


Where

$$
\begin{aligned}
X & =\frac{\bar{D}_{\mathrm{r}}}{D_{\mathrm{r}}}=\left[\frac{4}{\pi} \iint \mathrm{d} \Omega \mathrm{d} \Omega^{\prime} f(\boldsymbol{u} ; t) f\left(\boldsymbol{u}^{\prime} ; t\right) \sin \left(\boldsymbol{u}, \boldsymbol{u}^{\prime}\right)\right]^{-2} \\
& =\left[1-8 \pi \sum_{n=1}^{\infty} \sum_{m=0}^{2 n}\left(\frac{2 n-1}{2 n+2}\right)\left[\frac{(2 n-3) ! !}{(2 n) ! !}\right]^{2}\left|b_{2 n, m}\right|^{2}\right]^{-2}
\end{aligned}
$$

The explicit form of the matrix element $\left\langle l, m|\hat{\Gamma}| l^{\prime}, m^{\prime}\right\rangle$ is listed in ref 9 . From the normalization condition

$$
\int f(\boldsymbol{u}, t) \mathrm{d} \Omega=1
$$

$b_{00}$ is determined as

$$
b_{00}=1 / \sqrt{4 \pi}
$$

Equation 49 was solved numerically by truncating $b_{l m}$ with $l \geqq l^{*}\left(l^{*}\right.$ was taken as $\left.12-16\right)$. The three stress components are related to $b_{2 \mathrm{~m}}$ as

$$
\begin{aligned}
\sigma_{12} & =\sqrt{\frac{12 \pi}{5}} c k_{\mathrm{B}} T b_{21} \\
N_{1} & =\sqrt{\frac{12 \pi}{5}} c k_{\mathrm{B}} T\left(b_{22}-\sqrt{3} b_{20}\right) \\
N_{2} & =\sqrt{\frac{12 \pi}{5}} c k_{\mathrm{B}} T\left(b_{22}+\sqrt{3} b_{20}\right)
\end{aligned}
$$

Stress Growth in the Starts of Steady-State Flow

Suppose that a shear flow with a constant shear rate $\kappa$ starts at time $t=0$, i.e.,

$$
\kappa(t)= \begin{cases}0 & \text { for } t<0 \\ \kappa(=\text { constant }) & \text { for } t>0\end{cases}
$$

We discuss the steady stress in terms of the stressgrowth functions:

$$
\begin{aligned}
\sigma_{12}(t) & =\eta^{+}(\kappa ; t) \kappa \\
\sigma_{11}(t)-\sigma_{22}(t) & =\Psi_{1}^{+}(\kappa ; t) \kappa^{2} \\
\sigma_{22}(t)-\sigma_{33}(t) & =\Psi_{2}^{+}(\kappa ; t) \kappa^{2}
\end{aligned}
$$

These functions are obtained by solving eq 49 numerically with initial condition $b_{l m}=0(l \neq 0)$ at $t=0$. The growth of the shear stress is shown in Figure 6, where the function is normalized by the steady-state value shown in Figure 4.

The behaviour of the stress-growth curves is sensitive to the magnitude of $\kappa$. When $\kappa$ is small, the

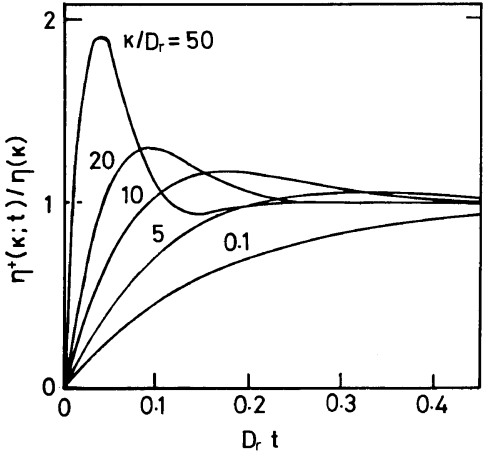

Figure 6. Shear stress when the shear flow starts at $t=0$ with constant shear rate $\kappa$.

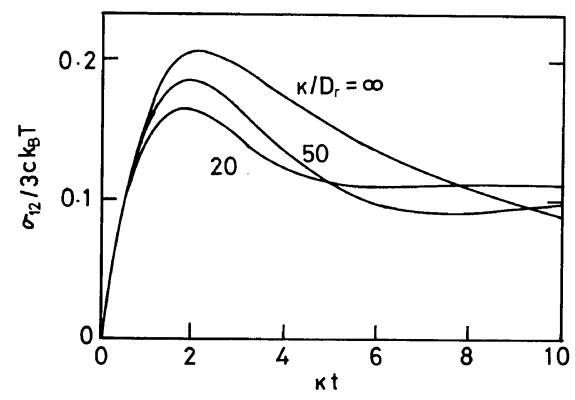

Figure 7. Plots of the shear stress component against shear strain.

stress increases monotonically with time, whereas when $\kappa$ is large enough, it shows a maximum before reaching the steady-state value. These phenomena are known in flexible polymers as the "stress overshoot." Figure 7 shows another plot of the results in Figure 6. Here the shear stress $\sigma_{12}$, is plotted against the shear strain $\gamma(=\kappa t)$. Let $t^{*}$ be the time at which the shear stress takes on a maximum. As observed in Figure 7, the product of $\kappa$ and $t^{*}$ is nearly independent of $\kappa$ and $D_{\mathrm{r}}$.

When $\kappa / D_{\mathrm{r}}$ is infinity, the stress tensor is given by eq 30 . The deformation tensor $\boldsymbol{E}$ is now represented by

$$
\boldsymbol{E}=\left[\begin{array}{lll}
1 & \gamma & 0 \\
0 & 1 & 0 \\
0 & 0 & 1
\end{array}\right]
$$

From eq 28 , the shear stress $\sigma_{12}$ becomes

$$
\sigma_{12}=\frac{3 c k_{\mathrm{B}} T}{2 \gamma} \int_{0}^{1} \mathrm{~d} x\left[1+\frac{x^{2} \gamma^{2}-1}{\sqrt{x^{4}\left(\gamma^{4}+4 \gamma^{2}\right)-2 \gamma^{2} x^{2}+1}}\right]
$$




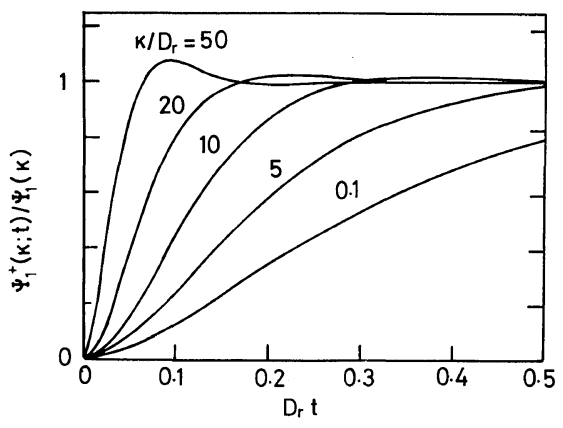

Figure 8. First normal stress coefficient when a shear flow starts at $t=0$ with shear rate $\kappa$.

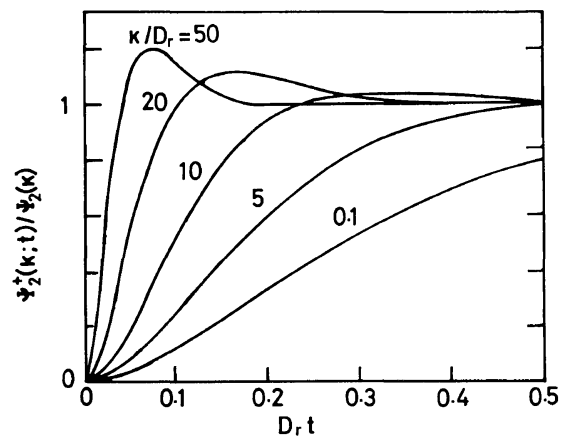

Figure 9. Second normal stress coefficient when the shear flow starts at $t=0$ with shear rate $\kappa$.

which is shown in the curve denoted by $\infty$ in Figure 9. Note that the curve has a maximum at a certain strain because the stress first increases with strain, but decreases as the rods align in the direction of the $x_{1}$ axis for a large deformation.

In the present model, the stress overshoot is qualitatively explained as follows: if $\kappa / D_{\mathrm{r}} \gtrsim 1$, the above stress peak still survives, but if $\kappa / D_{\mathrm{r}} \lesssim 1$, the peak is smeared out by the Brownian motion of the rods. This also explains the fact that $\kappa t^{*}$ is almost independent of $\kappa$ and $D_{\mathrm{r}}$. Figures 8 and 9 show the stress growth of first and second normal stress differences respectively. The stress overshoot is not so pronounced as in the shear stress.

\section{Stress Relaxation Following Cessation of the Steady Flow}

When the steady flow is suddenly stopped at time $t=0$, the stress decays with time. The development of shear rate is

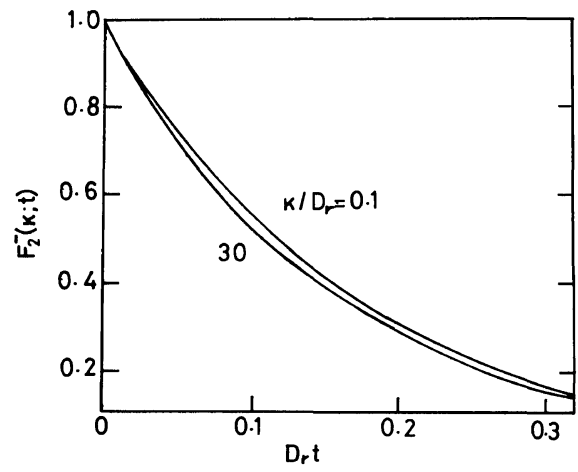

Figure 10. Relaxation function $F_{2}^{-}(\kappa ; t)$ when the shear flow, with shear rate $\kappa$, is stopped at $t=0$.

$$
\kappa(t)= \begin{cases}\kappa(=\text { constant }) & \text { for } t<0 \\ 0 & \text { for } t>0\end{cases}
$$

We introduce the stress-relaxation functions:

$$
\begin{aligned}
\sigma_{12}(t) & =\eta^{-}(\kappa ; t) \kappa \\
\sigma_{11}(t)-\sigma_{22}(t) & =\Psi_{1}^{-}(\kappa ; t) \kappa^{2} \\
\sigma_{22}(t)-\sigma_{33}(t) & =\Psi_{2}^{-}(\kappa ; t) \kappa^{2}
\end{aligned}
$$

In this case, eq 49 becomes

$$
\frac{\mathrm{d} b_{l m}}{\mathrm{~d} t}=-D_{\mathrm{r}} l(l+1) X b_{l m}
$$

Let $b_{l m}^{0}(\kappa)$ be the value of $b_{l m}$ in the steady state. The solution of eq 60 then takes on the form:

$$
b_{l m}(\kappa ; t)=b_{l m}^{0}(\kappa) F_{l}^{-}(\kappa ; t)
$$

where the function $F_{l}^{-}$depends on $l$ but not on $m$. Thus the relaxation curves $\eta^{-}, \Psi_{1}^{-}$, and $\Psi_{2}^{-}$have the same time-dependence:

$$
\begin{aligned}
\eta^{-}(\kappa ; t) / \eta(\kappa) & =\Psi_{1}^{-}(\kappa ; t) / \Psi_{1}(\kappa) \\
& =\Psi_{2}(\kappa ; t) / \Psi_{2}(\kappa)=F_{2}^{-}(\kappa ; t)
\end{aligned}
$$

When $\kappa$ is small enough, i.e., $\kappa / D_{\mathrm{r}} \lesssim 1$, the relaxation function is approximated as

$$
F^{-}(\kappa ; t) \approx \exp \left(-6 D_{\mathrm{r}} t\right)
$$

because, in this case, $X \approx 1$. For large values of $\kappa$, eq 60 was solved numerically with initial condition $b_{l m}=b_{l m}^{0}(\kappa)$ at $t=0$. As shown in Figure 10, the initial decay occurs faster for large $\kappa$ than for small $\kappa$. This is because, as $\kappa$ increases, the effective diffusion coefficient $\bar{D}_{\mathrm{r}}$ increases. 


\section{APPENDIX}

Equation 13 can be rewritten as

$\bar{\eta}(\dot{\varepsilon}) / \bar{\eta}(0)$

$$
=\frac{1}{2 \alpha}\left\{\frac{3}{\bar{\alpha}}\left[\left(\int_{0}^{1} \exp \left(-\bar{\alpha}\left(1-\zeta^{2}\right) / 2\right) \mathrm{d} \zeta\right)^{-1}-1\right]-1\right\}
$$

For $\alpha \gg 1$, exp $\left[-\bar{\alpha}\left(1-\zeta^{2}\right) / 2\right]$ has a very sharp peak at $\zeta \approx 1$. Replacing $\zeta$ by $1-s$, the integral in eq A 1 becomes

$$
\int_{0}^{1} \exp \left[-\bar{\alpha}\left(1-\zeta^{2}\right) / 2\right] \mathrm{d} \zeta=\int_{0}^{1} \exp \left[-\bar{\alpha} s+\bar{\alpha} s^{2} / 2\right] \mathrm{d} s
$$

Since the integrand of the right-hand side has a very sharp peak at $s \cong 0$, the integral is evaluated as

$$
\begin{aligned}
\int_{0}^{1} \exp \left[-\bar{\alpha} s+\bar{\alpha} s^{2} / 2\right] \mathrm{d} s \\
\quad \approx \frac{1}{\bar{\alpha}} \int_{0}^{\infty} \exp \left(-\xi+\xi^{2} / 2 \bar{\alpha}\right) \mathrm{d} \xi \\
=\frac{1}{\bar{\alpha}} \sum_{n=0}^{\infty} \frac{1}{n !} \int_{0}^{\infty} \mathrm{e}^{-\xi\left(\frac{\xi^{2}}{2 \bar{\alpha}}\right) \mathrm{d} \xi} \\
=\frac{1}{\bar{\alpha}} \sum_{n=0}^{\infty} \frac{(2 n+1) !}{(2 n) ! !} \frac{1}{\alpha^{n+1}} \\
=\frac{1}{\bar{\alpha}}+\frac{1}{\bar{\alpha}^{2}}+\frac{15}{\bar{\alpha}^{3}}+\frac{105}{\bar{\alpha}^{4}}+\cdots
\end{aligned}
$$

Then from eq A 1 and A 3 , we obtain eq 18. The relation between $\alpha$ and $\bar{\alpha}$ can be calculated as follows. Because the distribution function $f(\boldsymbol{u})$ has a very sharp peak around the polar axis of $\theta=0$, $\sin \left(\boldsymbol{u}, \boldsymbol{u}^{\prime}\right)$ can be replaced as

$$
\sin \left(\boldsymbol{u} ; \boldsymbol{u}^{\prime}\right) \approx\left(\boldsymbol{u}, \boldsymbol{u}^{\prime}\right) \approx \sqrt{\theta^{2}+\theta^{\prime 2}-2 \theta \theta^{\prime} \cos \left(\varphi-\varphi^{\prime}\right)}
$$

Therefore from eq 8 and A 4 we obtain

$$
\bar{\alpha} \approx \mathscr{T} \sqrt{\alpha}
$$

with

$$
\begin{aligned}
\mathscr{T}= & \frac{16}{\pi^{2}} \int_{0}^{2 \pi} \mathrm{d} \varphi \int_{0}^{\infty} \xi \mathrm{d} \xi \int_{0}^{\infty} \eta \mathrm{d} \eta \mathrm{e}^{-\left(\xi^{2}+\eta^{2}\right)} \\
& \times \sqrt{\xi^{2}+\eta^{2}-2 \xi \eta \cos \varphi} \\
= & 2.6010
\end{aligned}
$$

\section{REFERENCES}

1. W. B. Black, "Stiff Chain Aromatic Polymer Solutions, Melts and Fibers," in "Flow Induced Crystallization in Polymer Systems," R. L. Millar, Ed., Gordon and Breach, New York, N.Y., 1977.

2. S. P. Papkov, V. G. Kulichkhin, V. D. Kalmykov, and A. Y. Malkin, J. Polym. Sci., 12, 1753 (1974).

3. G. D. Baird and R. L. Ballman, J. Rheol., 23, 505 (1979).

4. T. E. Helminiak and G. C. Berry, J. Polym. Sci., Polym. Symp., No. 65, 107 (1978).

5. N. Ookubo, M. Komatsubara, H. Nakajima, and Y. Wada, Biopolymers, 15, 929 (1976).

6. T. Asada, H. Muramatsu, and S. Onogi, Nihon Reorojī Gakkaishi, 6, 130 (1978).

7. M. Doi, J. Phys. (Paris), 36, 607 (1975).

8. M. Doi and S. F. Edwards, J. Chem. Soc., Faraday Trans., 2, 74, 568 (1978).

9. M. Doi and. S. F. Edwards, J. Chem. Soc., Faraday Trans., 2, 74, 918 (1978).

10. R. B. Bird, R. C. Armstrong, and O. Hassager, "Dynamics of Polymeric Liquids," Vol. 1, John Wiley \& Sons, New York, N.Y., 1977. 